Revista de Matemática: Teoría y Aplicaciones 2007 14(1) : 45-64

CIMPA - UCR - CCSS ISSN: 1409-2433

\title{
VALORES PROPIOS ASOCIADOS AL OPERADOR DE HILL UNIDIMENSIONAL. CASO GENERAL
}

\author{
Santiago Cambronero* Marco Alfaro $^{\dagger}$
}

Recibido/Received: 30 Nov 2006 - Aceptado/Accepted: 31 Jan 2007

\begin{abstract}
Resumen
Estudiamos la ecuación de Hill con condiciones generales de frontera y potencial ruido blanco. En [3] los autores resuelven el problema con condiciones de Dirichlet, dejando el caso general sin resolver. El problema reviste importancia tanto desde un punto de vista teórico como aplicado, dado el rango de aplicaciones del tema. Siguiendo a [6], hacemos una descripción del movimiento browniano estándar y sus propiedades básicas, para luego introducir la ecuación que será nuestro objeto de estudio. Posteriormente hacemos el estudio del comportamiento asintótico de los valores propios.
\end{abstract}

Palabras clave: Operador de Hill, condiciones de frontera, movimiento Browniano, valores propios.

\begin{abstract}
We study Hill's equation with general boundary conditions and white noise potential. In [3] they solve the problem with Dirichlet conditions, leaving the general case unresolved. The problem is important both from the theoretical and the applied point of view, given the range of applications of the subject. Following [6], we give a description of the standard brownian motion process, and mention some of its basic properties. We then introduce the equation to be studied, the remaining of the work being devoted to the analysis of the asymptotic of eigenvalues.
\end{abstract}

Keywords: Hill's operator, boundary conditions, Brownian motion, eigenvalues.

Mathematics Subject Classification: 60H40, 60J65, 60H25, 47B80, 60H10, 34F05, $60 \mathrm{~K} 40$.

\footnotetext{
${ }^{*}$ CIMPA, Escuela de Matemática, Universidad de Costa Rica, 2060 San José, Costa Rica. E-Mail: scambro@emate.ucr.ac.cr

†Escuela de Matemática, Universidad de Costa Rica, 2060 San José, Costa Rica. E-Mail: malfaro28@hotmail.com
} 


\section{Preliminares}

Presentamos en esta sección, las herramientas básicas que nos permitirán establecer con claridad el problema central del trabajo. Comenzamos con la definición del movimiento browniano estándar.

\subsection{Movimiento Browniano}

Un movimiento browniano estándar, es un proceso estocástico $\left(b_{t}\right)_{t \geq 0}$ con caminos continuos, tal que $P\left[b_{0}=0\right]=1$, y tal que los incrementos

$$
b\left(t_{1}\right)-b\left(t_{0}\right), \ldots, b\left(t_{n}\right)-b\left(t_{n-1}\right)
$$

son variables independientes y normalmente distribuidas (para $t_{n}>t_{n-1}>\cdots>t_{0}=0$ ), con media 0 y varianza $t_{i}-t_{i-1}$. Nótese que, por la independencia de los incrementos se tiene

$$
E[b(t) b(s)]=\min (t, s) .
$$

Un movimiento browniano estándar, se puede definir también como una familia gaussiana $\left(b_{t}\right)_{t \geq 0}$, con caminos continuos, tal que $P\left[b_{0}=0\right]=1, E b_{t}=0, \mathrm{y}$

$$
\operatorname{cov}(b(t), b(s))=\min (t, s), \forall t, s \geq 0
$$

El cambio de variables $y_{i}=x_{i}-x_{i-1}$ permite obtener las distribuciones finito - dimensionales

$$
P\left[b_{t_{1}} \in A_{1}, \ldots, b_{t_{k}} \in A_{k}\right]=\int_{A_{1}} \ldots \int_{A_{k}} \prod_{i=1}^{k} \frac{e^{-\left(x_{i}-x_{i-1}\right)^{2} / 2\left(t_{i}-t_{i-1}\right)}}{\sqrt{2 \pi\left(t_{i}-t_{i-1}\right)}} d x_{i}
$$

para $0=t_{0}<t_{1} \cdots<t_{k}, A_{1}, \ldots, A_{k} \in \mathcal{B}_{\mathbb{R}}, x_{0}=0^{1}$. En particular, para $t>s$ se tiene

$$
P[b(t) \in A, b(s) \in B]=\int_{B}\left[\int_{A} \frac{e^{-(y-x)^{2} / 2(t-s)}}{\sqrt{2 \pi(t-s)}} d y\right] \frac{e^{-x^{2} / 2 s}}{\sqrt{2 \pi s}} d x,
$$

para todo $B \in \mathcal{B}_{\mathbb{R}}$, y por lo tanto

$$
P[b(t) \in A \mid b(s)=x]=\int_{A} \frac{e^{-(y-x)^{2} / 2(t-s)}}{\sqrt{2 \pi(t-s)}} d y=\int_{A} p(t-s ; x, y) d y
$$

donde identificamos la densidad de transición browniana estándar

$$
p(\tau ; x, y)=\frac{1}{\sqrt{2 \pi \tau}} e^{-(y-x)^{2} / 2 \tau} .
$$

\footnotetext{
${ }^{1}$ Aquí $\mathcal{B}_{\mathbb{R}}$ denota la $\sigma$-álgebra de Borelianos de $\mathbb{R}$.
} 


\subsubsection{Propiedades básicas}

A continuación enunciaremos una serie de propiedades del movimiento browniano, que nos serán de gran utilidad en el presente trabajo.

Lema 1.1 (Propiedad de Markov) Dado un movimiento browniano b(t) y dado $s>0$, el proceso $\left(X_{t}\right)_{t \geq 0}$ definido por $X_{t}=b(t+s)-b(s)$, es también un movimiento browniano, el cual es independiente de $\mathcal{F}_{s}=\sigma(b(\tau): \tau \leq s)$.

Lema $1.2 b^{2}(t)-t$ es una martingala.

\subsubsection{Desigualdades con martingalas}

En el desarrollo del presente trabajo, necesitaremos las siguientes desigualdades sobre martingalas, cuyas demostraciones pueden ser halladas en la literatura.

Si $\left(X_{t}\right)_{t \geq 0}$ es una submartingala con caminos continuos, entonces para $\lambda>0, T>0$, se tiene

$$
P\left[\max _{0 \leq t \leq T} X_{t} \geq \lambda\right] \leq \lambda^{-1} E X_{T}^{+} .
$$

En particular, si $\left(X_{t}\right)_{t \geq 0}$ es una martingala y $p \geq 1$, entonces aplicando lo anterior a la submartingala $\left(\left|X_{t}\right|^{p}\right)_{t \geq 0}$, y se tiene

$$
P\left[\max _{0 \leq t \leq T}\left|X_{t}\right| \geq \lambda\right] \leq \lambda^{-p} E\left|X_{T}\right|^{p} .
$$

El lector puede consultar [2], [8], [9] y [12] para las demostraciones.

\subsubsection{Otras propiedades}

Para las demostraciones de las propiedades siguientes, puede consultarse [2], [8], [9] y [12].

1. El movimiento browniano satisface la ley fuerte de grandes números:

$$
\frac{b(t)}{t} \rightarrow 0, \text { cuando } t \rightarrow \infty, \text { casi seguramente. }
$$

2. Dado un movimiento browniano $b(t)$, los procesos $X_{t}=c b\left(t / c^{2}\right)$ y $Y_{t}=t b(1 / t)$ son también movimientos brownianos. Para probar esto basta con observar que

$$
E\left[X_{t} X_{s}\right]=E\left[Y_{t} Y_{s}\right]=\min (t, s) .
$$

En el segundo caso la continuidad en el origen no es evidente, pero es consecuencia de la propiedad 1.

3. El proceso $-b(t): t \geq 0$ es un browniano.

4. Los caminos brownianos son derivables en ningún punto. En otras palabras,

$$
P[b: b \text { es derivable en al menos un } t \geq 0]=0 .
$$


5. (Ley del logaritmo iterado) El movimiento browniano satisface

$$
P\left[\limsup _{t \rightarrow 0^{+}} \frac{b(t)}{\sqrt{2 t \ln \ln \frac{1}{t}}}=1\right]=P\left[\limsup _{t \rightarrow \infty} \frac{b(t)}{\sqrt{2 t \ln \ln t}}=1\right]=1 .
$$

6. (Módulo de continuidad de Lévy) El movimiento browniano satisface

$$
P\left[\lim _{t \rightarrow 0} \sup _{\left|t_{1}-t_{2}\right|=t} \frac{\left|b\left(t_{1}\right)-b\left(t_{2}\right)\right|}{\sqrt{2 t \ln \frac{1}{t}}}=1\right]=1 \text {. }
$$

En particular, si

$$
H_{\alpha}=\{b: b \text { es Hölder continua con parámetro } \alpha\},
$$

entonces

$$
P\left(H_{\alpha}\right)= \begin{cases}1 & \text { si } \alpha<\frac{1}{2} \\ 0 & \text { si } \alpha \geq \frac{1}{2}\end{cases}
$$

\subsection{Ruido blanco}

Aunque el movimiento Browniano es derivable en ninguna parte, se podría pensar en un proceso generalizado $q(t), t \geq 0$, cuyos caminos sean las derivadas (en un sentido formal) de los caminos del movimiento browniano. En este sentido el proceso $q(t)$ sería un ruido blanco si posee las distribuciones finito-dimensionales

$$
\operatorname{Pr}\left[\frac{1}{h} \int_{0}^{h} q_{1} \in A_{1}, \ldots, \frac{1}{h} \int_{(1-n) h}^{1} q_{n} \in A_{n}\right]=\int_{A_{1}} \ldots \int_{A_{n}} \prod_{i=1}^{n} \frac{e^{-h q_{i}^{2} / 2}}{\sqrt{2 \pi / h}} d q_{i}
$$

con $h n=1$.

Debido a que los caminos del movimiento browniano son Hölder-continuos de parámetro menor que $\frac{1}{2}$, los caminos del ruido blanco son elementos de espacios de Sobolev $H^{\varepsilon}$, con $\varepsilon<-\frac{1}{2}$. La construcción rigurosa se puede realizar, pero no es uno de los objetivos de este trabajo. De hecho, el único punto en que requerimos el ruido blanco es en el planteo de de la ecuación de Hill. Vía integración formal por partes, esta ecuación se interpreta fácilmente usando el movimiento browniano estándar, y su tratamiento posterior no requiere mención alguna del ruido blanco como proceso.

Ahora, formalmente si se requiere que el ruido blanco sea periódico, es decir, que $q(x+1)=$ $q(x)$, deberá tenerse

$$
b(x+1)-b(1)=b(x),
$$

así que debemos condicionar el movimiento browniano para que cumpla esta condición. La manera más fácil de lograr esto es tomar un movimiento browniano estándar en [0,1], y luego extenderlo en forma recursiva mediante

$$
b(x)=b(1)+b(x+1), \quad x \geq 1 .
$$

Otra manera de lograr esto es vía distribuciones finito-dimensionales. 


\section{Planteamiento del problema}

Como hemos mencionado, nuestro objetivo es el estudio de los valores propios de la ecuación de Hill

$$
-y^{\prime \prime}+q y=\lambda y
$$

donde el potencial $q$ es ruido blanco. Una solución de esta ecuación es una función $\varphi$ de clase $\mathcal{C}^{1}$ que satisface la versión integral

$$
-\varphi^{\prime}(x)+\varphi^{\prime}(0)+\int_{0}^{x} \varphi(t) d b(t)=\lambda \int_{0}^{x} \varphi(t) d t,
$$

donde $b$ es el correspondiente movimiento browniano en $(\Omega, \mathcal{B}, P)$, y la integral de la izquierda se define como

$$
\varphi(x) b(x)-\int_{0}^{x} b(t) \varphi^{\prime}(t) d t
$$

Se considera el problema de valores de frontera:

$$
\left\{\begin{array}{l}
-y^{\prime \prime}+q y=\lambda y \\
y(0)=h y(0), y^{\prime}(1)=-H y(1) .
\end{array}\right.
$$

Para el análisis que realizaremos, es importante considerar la solución $\varphi=\varphi(x, \lambda)$ del problema:

la cual satisface:

$$
\left\{\begin{array}{l}
-y^{\prime \prime}+q y=\lambda y \\
y^{\prime}(0)=1, \quad y^{\prime}(0)=h
\end{array}\right.
$$

$$
\varphi(x)=\varphi_{0}(x)+\int_{0}^{x} \frac{\operatorname{sen} \sqrt{\lambda}(x-t)}{\sqrt{\lambda}} q(t) \varphi(t) d t,
$$

$\operatorname{con} \varphi_{0}(x)=\frac{h}{\sqrt{\lambda}} \operatorname{sen} \sqrt{\lambda} x+\cos \sqrt{\lambda} x$. Si denotamos

$$
R_{0}(x)=\int_{0}^{x} \frac{\operatorname{sen} \sqrt{\lambda}(x-t)}{\sqrt{\lambda}} q(t) \varphi(t) d t
$$

se tiene $\varphi(x)=\varphi_{0}(x)+R_{0}(x)$, y entonces, como en [3] se obtiene

$$
\begin{aligned}
\varphi(x) & =\varphi_{0}(x)+\int_{0}^{x} \frac{\operatorname{sen} \sqrt{\lambda}(x-t)}{\sqrt{\lambda}} q(t)\left[\varphi_{0}(t)+R_{0}(t)\right] d t \\
& =\varphi_{0}(x)+\varphi_{1}(x)+R_{1}(x)
\end{aligned}
$$

donde

$$
\begin{aligned}
& \varphi_{1}(x)=\int_{0}^{x} \frac{\operatorname{sen} \sqrt{\lambda}(x-t)}{\sqrt{\lambda}} q(t) \varphi_{0}(t) d t \\
& R_{1}(x)=\int_{0}^{x} \frac{\operatorname{sen} \sqrt{\lambda}(x-t)}{\sqrt{\lambda}} q(t) R_{0}(t) d t .
\end{aligned}
$$


Iterando este proceso se obtiene

$$
\varphi(x)=\varphi_{0}(x)+\varphi_{1}(x)+\ldots+\varphi_{n}(x)+R_{n}(x),
$$

donde $\varphi_{n}(x)$ se define inductivamente por:

$$
\varphi_{n+1}(x)=\int_{0}^{x} \frac{\operatorname{sen} \sqrt{\lambda}(x-t)}{\sqrt{\lambda}} q(t) \varphi_{n}(t) d t
$$

y de la misma manera

$$
R_{n+1}(x)=\int_{0}^{x} \frac{\operatorname{sen} \sqrt{\lambda}(x-t)}{\sqrt{\lambda}} q(t) R_{n}(t) d t .
$$

En adelante denotaremos

$$
S_{\lambda}(x, t)=\frac{\operatorname{sen} \sqrt{\lambda}(x-t)}{\sqrt{\lambda}}, \quad C_{\lambda}(x, t)=\frac{\partial S_{\lambda}(x, t)}{\partial x}=\cos \sqrt{\lambda}(x-t) .
$$

Se tiene entonces

$$
\varphi_{n+1}(x)=\int_{0}^{x} S_{\lambda}(x, t) q(t) \varphi_{n}(t) d t
$$

y es fácil verificar que

$$
\varphi_{n+1}^{\prime}(x)=\int_{0}^{x} C_{\lambda}(x, t) q(t) \varphi_{n}(t) d t,
$$

dado que $S_{\lambda}(x, x) \equiv 0$. Integrando formalmente por partes se obtiene

$$
\begin{aligned}
\varphi_{n+1}(x) & =\int_{0}^{x} S_{\lambda}(x, t) \varphi_{n}(t) d b(t) \\
& =\int_{0}^{x}\left[C_{\lambda}(x, t) \varphi_{n}(t)-S_{\lambda}(x, t) \varphi_{n}^{\prime}(t)\right] b(t) d t
\end{aligned}
$$

y similarmente

$$
\begin{aligned}
\varphi_{n+1}^{\prime}(x) & =\int_{0}^{x} C_{\lambda}(x, t) \varphi_{n}(t) d b(t) \\
& =\varphi_{n}(x) b(x)-\int_{0}^{x}\left[\lambda S(x, t) \varphi_{n}(t)+C_{\lambda}(x, t) \varphi_{n}^{\prime}(t)\right] b(t) d t
\end{aligned}
$$

Si se denota $c=|b|=\max _{0 \leq t \leq 1}|b(t)|$, se obtiene que, para $\lambda$ real,

$$
\left|\varphi_{n+1}(x)\right| \leq c \int_{0}^{x}\left(\left|\varphi_{n}(t)\right|+\frac{1}{\sqrt{\lambda}}\left|\varphi_{n}^{\prime}(t)\right|\right) d t
$$

y

$$
\left|\varphi_{n+1}^{\prime}(x)\right| \leq c \int_{0}^{x}\left(\sqrt{\lambda}\left|\varphi_{n}(t)\right|+\left|\varphi_{n}^{\prime}(t)\right|\right) d t+c\left|\varphi_{n}(x)\right|,
$$


así que

$$
\frac{1}{\sqrt{\lambda}}\left|\varphi_{n+1}^{\prime}(x)\right| \leq c \int_{0}^{x}\left(\left|\varphi_{n}(t)\right|+\frac{1}{\sqrt{\lambda}}\left|\varphi_{n}^{\prime}(t)\right|\right) d t+\frac{c}{\sqrt{\lambda}}\left|\varphi_{n}(x)\right| .
$$

Para manipular mejor estas desigualdades, definamos

$$
f_{n}(x)=\left|\varphi_{n}(x)\right|, \quad g_{n}(x)=\frac{1}{\sqrt{\lambda}}\left|\varphi_{n}^{\prime}(x)\right| .
$$

Lo anterior nos dice entonces que

$$
\begin{aligned}
& 0 \leq f_{n}(x) \leq c \int_{0}^{x}\left(f_{n}(t)+g_{n}(t)\right) d t \\
& 0 \leq g_{n}(x) \leq c \int_{0}^{x}\left(f_{n}(t)+g_{n}(t)\right) d t+\frac{c}{\mu} f_{n}(x),
\end{aligned}
$$

donde $\mu=\sqrt{\lambda}$. Entonces se satisfacen las condiciones del Lema 2 de [3], gracias al cual se obtiene, para $\mu>2 c$ y $x \in[0,1]$,

$$
\sum_{n=0}^{\infty} h_{n}(x) \leq 2 e^{4 c}\left|h_{0}(x)\right|,
$$

donde $h_{n}=\max \left(f_{n}, g_{n}\right)$. Esto demuestra en particular que la serie $\sum_{n=0}^{\infty} \varphi_{n}(x)$ converge uniformemente en $[0,1]$, lo mismo que $\sum_{n=0}^{\infty} \varphi_{n}^{\prime}(x)$. Denotemos $\varphi(x)=\sum_{n=0}^{\infty} \varphi_{n}(x)$.

Ahora, dado que

$$
\begin{aligned}
\sum_{k=0}^{n} \varphi_{k}(x)= & \varphi_{0}(x)+\sum_{k=0}^{n-1} \varphi_{k+1}(x) \\
= & \varphi_{0}(x)+\sum_{k=0}^{n-1} \int_{0}^{x}\left[C_{\lambda}(x, t) \varphi_{k}(t)-S_{\lambda}(x, t) \varphi_{k}^{\prime}(t)\right] b(t) d t \\
= & \varphi_{0}(x)+ \\
& \int_{0}^{x}\left(C_{\lambda}(x, t) \sum_{k=0}^{n-1} \varphi_{k}(t) b(t)-S_{\lambda}(x, t) \sum_{k=0}^{n-1} \varphi_{k}^{\prime}(t) b(t)\right) d t
\end{aligned}
$$

por convergencia uniforme se obtiene

$$
\varphi(x)=\varphi_{0}(x)+\int_{0}^{x}\left[C_{\lambda}(x, t) \varphi(t)-S_{\lambda}(x, t) \varphi^{\prime}(t)\right] b(t) d t,
$$

y por lo tanto $\varphi(x)$ es la solución del problema (4).

Teorema 1 Para $\lambda>4|b|^{2}$, la serie $\sum_{n=0}^{\infty} \varphi_{n}(x)$ definida anteriormente, converge a una solución $\varphi$ del problema (4). Más específicamente, $\varphi$ satisface:

$$
\left\{\begin{array}{l}
-\varphi^{\prime \prime}+q \varphi=\lambda \varphi \\
\varphi(0)=1, \varphi^{\prime}(0)=h
\end{array}\right.
$$

Además, la convergencia de $\sum_{n=0}^{\infty} \varphi_{n}(x)$ y $\sum_{n=0}^{\infty} \varphi_{n}^{\prime}(x)$ es uniforme en $[0,1]$. 


\subsection{Comportamiento asintótico de los valores propios}

El Lema 2 de [3] también proporciona una cota para el error $R_{n}(x)$; en efecto, tomando ahora

$$
f_{k}(x)=\left|\varphi_{n+k}(x)\right|, g_{k}(x)=\frac{1}{\mu}\left|\varphi_{n+k}^{\prime}(x)\right|
$$

se obtiene

$$
\left|R_{n}(x)\right| \leq \sum_{k=n+1}^{\infty}\left|\varphi_{k}(x)\right| \leq 2 e^{4|b|} \max \left(\left|\varphi_{n+1}(x)\right|, \frac{1}{\mu}\left|\varphi_{n+1}^{\prime}(x)\right|\right) .
$$

Corolario 1 El resto $R_{n}$ satisface:

$$
\left|R_{n}(x)\right| \leq 2 e^{4|b|} \max \left(\left|\varphi_{n+1}(x)\right|, \frac{1}{\mu}\left|\varphi_{n+1}^{\prime}(x)\right|\right)
$$

$y$

$$
\left|R_{n}^{\prime}(x)\right| \leq 2 \mu e^{4|b|} \max \left(\left|\varphi_{n+1}(x)\right|, \frac{1}{\mu}\left|\varphi_{n+1}^{\prime}(x)\right|\right)
$$

Como veremos más adelante, se obtienen entonces cotas para $\varphi_{n+1}(x)$ y $\frac{1}{\sqrt{\lambda}} \varphi_{n+1}^{\prime}(x)$ del mismo orden, lo que determina el orden de $R_{n}(x)$. El resto del trabajo, lo dedicaremos a demostrar el siguiente teorema.

Teorema 2 (Teorema principal) La sucesión de valores propios del problema (3) tiene el siguiente desarrollo asintótico:

$$
\lambda_{n}=n^{2} \pi^{2}+2 h+2 H+b(1)-\frac{1}{2} \int_{0}^{1} b^{2}(t) d t+O\left(n^{-1+}\right) .
$$

\section{Demostración del teorema principal}

Colocando $\mu=\sqrt{\lambda}$, con las notaciones introducidas arriba, se tiene que

$$
\begin{aligned}
\varphi_{1}(x)= & \int_{0}^{x}\left[C_{\lambda}(x, t) \varphi_{0}(t)-S_{\lambda}(x, t) \varphi_{0}^{\prime}(t)\right] b(t) d t \\
= & h \int_{0}^{x}\left(\cos \mu(x-t) \frac{\operatorname{sen} \mu t}{\mu}-\frac{\operatorname{sen} \mu(x-t)}{\mu} \cos \mu t\right) b(t) d t \\
& +\int_{0}^{x}(\cos \mu(x-t) \cos \mu t+\operatorname{sen} \mu(x-t) \operatorname{sen} \mu t) b(t) d t \\
= & \frac{h}{\mu} \int_{0}^{x} \operatorname{sen}[\mu(2 t-x)] b(t) d t+\int_{0}^{x} \cos [\mu(2 t-x)] b(t) d t \\
= & \frac{h}{\mu} \operatorname{Im}\left(e^{-i \mu x} I_{1}(x)\right)+\operatorname{Re}\left(e^{-i \mu x} I_{1}(x)\right)
\end{aligned}
$$


donde $I_{1}=\int_{0}^{x} b(t) e^{2 i \mu t} d t$. En [3] se demuestra que para casi todo camino browniano se tiene

$$
\left|I_{1}(x)\right| \leq 2 \mu^{-\frac{1}{2}}(\log \mu)^{\frac{1}{2}}, \quad \text { cuando } \mu \uparrow \infty .
$$

Por lo tanto, si además $\mu \geq|h|$, se deuce que

$$
\left|\varphi_{1}(x)\right| \leq \sqrt{2}\left|I_{1}(x)\right| \leq 3 \mu^{-\frac{1}{2}}(\log \mu)^{\frac{1}{2}} .
$$

Por otra parte, de (6) se sigue que

$$
\begin{aligned}
\varphi_{1}^{\prime}(t)=\left(\frac{h}{\mu} \operatorname{sen} \mu x+\cos \mu x\right) b(x)- & h \int_{0}^{x} \cos \mu x(2 t-x) b(t) d t \\
& +\mu \int_{0}^{x} \operatorname{sen} \mu(2 t-x) b(t) d t \\
= & -h \operatorname{Re}\left(e^{-i \mu x} I_{1}(x)\right)+\mu \operatorname{Im}\left(e^{-i \mu x} I_{1}(x)\right)+\left(\frac{h}{\mu} \operatorname{sen} \sqrt{\lambda} x+\cos \mu x\right) b(t),
\end{aligned}
$$

así que

$$
\begin{aligned}
\frac{1}{\mu}\left|\varphi_{1}^{\prime}(x)\right| & \leq \sqrt{2}\left|I_{1}(x)\right|+\left(\frac{|h|}{\mu^{2}}+\frac{1}{\mu}\right)|b| \\
& \leq 3 \mu^{-\frac{1}{2}}(\log \mu)^{\frac{1}{2}}, \quad \text { cuando } \mu \uparrow \infty .
\end{aligned}
$$

De lo anterior, y el corolario al Teorema 2, se obtiene

$$
\begin{aligned}
\varphi(x) & =\varphi_{0}(x)+R_{0}(x) \\
& =\frac{h}{\mu} \operatorname{sen} \mu x+\cos \mu x+O\left(\mu^{-\frac{1}{2}+}\right) \\
& =\cos \mu x+O\left(\mu^{-\frac{1}{2}+}\right),
\end{aligned}
$$

en donde $f(x)=O\left(n^{\alpha+}\right)$ significa que $f(x)=O\left(n^{\alpha+\varepsilon}\right)$, para todo $\varepsilon>0$, y análogamente para $O\left(n^{\alpha-}\right)$. Además se tiene

$$
\varphi^{\prime}(x)=-\mu \operatorname{sen} \mu x+O\left(\mu^{-\frac{1}{2}+}\right)
$$

Para que $\lambda$ sea valor propio, se necesita

$$
\varphi^{\prime}(1)=-H \varphi(1)
$$

de donde debemos tener

$$
\varphi^{\prime}(1)=-\mu \operatorname{sen} \mu+O\left(\mu^{\frac{1}{2}+}\right)=-H \cos \mu+O\left(\mu^{-\frac{1}{2}+}\right) .
$$


Esto nos permite concluir que

$$
\operatorname{sen} \mu=O\left(\mu^{-\frac{1}{2}+}\right), \quad \text { cuando } \mu \uparrow \infty .
$$

Por lo tanto, la sucesión de valores propios $\left(\lambda_{n}\right)$ viene dada asintóticamente por $\lambda_{n}=\mu_{n}^{2}$, donde $\mu_{n}=n \pi+\varepsilon_{n}$, con $\varepsilon_{n} \rightarrow 0$. De (7) se sigue que

$$
\operatorname{sen} \varepsilon_{n}=\varepsilon_{n}+O\left(\varepsilon_{n}^{3}\right)=O\left(\frac{1}{n^{\frac{1}{2}-}}\right),
$$

y entonces

$$
\varepsilon_{n}=O\left(n^{-\frac{1}{2}+}\right)
$$

Tenemos entonces que

$$
\varphi(x)=\varphi_{0}(x)+\varphi_{1}(x)+\ldots=\varphi_{0}(x)+R_{0}(x),
$$

donde el orden de $R_{0}$ está determinado por el orden de $\varphi_{1}$.

Ahora

$$
\varphi_{1}(x)=\frac{h}{\mu} \int_{0}^{x} \operatorname{sen} \mu(2 t-x) b(t) d t+\int_{0}^{x} \cos \mu(2 t-x) b(t) d t
$$

y

$$
\varphi_{0}(x)=\frac{h}{\mu} \operatorname{sen}(\mu x)+\cos (\mu x) .
$$

Nuevamente, como $\varphi^{\prime}(1)=-H \varphi(1)$, tenemos que,

$$
\varphi_{0}^{\prime}(1)+\varphi_{1}^{\prime}(1)=-H\left[\varphi_{0}(1)+\varphi_{1}(1)+O\left(\mu^{-1+}\right)\right]
$$

donde

$$
\begin{aligned}
\varphi_{0}^{\prime}(1) & =h \cos \mu-\mu \operatorname{sen} \mu \\
& =h \cos \left(n \pi+\varepsilon_{n}\right)-\left(n \pi+\varepsilon_{n}\right) \operatorname{sen}\left(n \pi+\varepsilon_{n}\right) \\
& =(-1)^{n}\left[h \cos \varepsilon_{n}-\left(n \pi+\varepsilon_{n}\right) \operatorname{sen} \varepsilon_{n}\right] \\
& =(-1)^{n}\left[h\left(1+O\left(\varepsilon_{n}^{2}\right)\right)-n \pi\left(1+\frac{\varepsilon_{n}}{n \pi}\right)\left(\varepsilon_{n}-\frac{\varepsilon_{n}^{3}}{6}+O\left(\varepsilon_{n}^{5}\right)\right)\right] \\
& =(-1)^{n}\left[h-n \pi \varepsilon_{n}+\frac{n \pi \varepsilon_{n}^{3}}{6}+O\left(n^{-1+}\right)\right] .
\end{aligned}
$$

Por otra parte,

$$
\begin{aligned}
\varphi_{1}^{\prime}(1)= & -h \int_{0}^{x} \cos \left[\left(n \pi+\varepsilon_{n}\right)(2 t-1)\right] b(t) d t+\frac{h}{n \pi+\varepsilon_{n}} \operatorname{sen}\left(n \pi+\varepsilon_{n}\right) b(1) \\
& +\left(n \pi+\varepsilon_{n}\right) \int_{0}^{x} \operatorname{sen}\left[\left(n \pi+\varepsilon_{n}\right)(2 t-1)\right] b(t) d t+\cos \left(n \pi+\varepsilon_{n}\right) b(1)
\end{aligned}
$$


Obsérvese que, por el Corolario 1 de [3], se obtiene que

$$
\int_{0}^{1} \cos \left[\left(n \pi+\varepsilon_{n}\right)(2 t-1)\right] b(t) d t=O\left(n^{-1+}\right),
$$

también

$$
\frac{h}{n \pi+\varepsilon_{n}} \operatorname{sen}\left(n \pi+\varepsilon_{n}\right) b(1)=O\left(n^{-1+}\right)
$$

y

$$
\cos \left(n \pi+\varepsilon_{n}\right) b(1)=(-1)^{n} b(1)+O\left(n^{-1+}\right) .
$$

Aplicando los Lemas 3-4 y el Corolario 1 de [3], y después de algunas simplificaciones se llega a

$$
\begin{aligned}
I_{n} & =\left(n \pi+\varepsilon_{n}\right) \int_{0}^{1} \operatorname{sen}\left[2 \pi n t-n \pi+\varepsilon_{n}(2 t-1)\right] b(t) d t \\
& =(-1)^{n}\left[n \pi Y_{n}-n \pi \varepsilon_{n} X_{n}+2 n \pi \varepsilon_{n} \widehat{X}_{n}\right]+O\left(n^{-1+}\right)
\end{aligned}
$$

donde $I_{n}=\left(n \pi+\varepsilon_{n}\right) \int_{0}^{1} \operatorname{sen}\left(\left(n \pi+\varepsilon_{n}\right)(2 t-1)\right) b(t) d t, \mathrm{y}$

$$
\begin{gathered}
Y_{n}=\int_{0}^{1} \operatorname{sen}(2 \pi n t) b(t) d t, X_{n}=\int_{0}^{1} \cos (2 \pi n t) b(t) d t \\
\widehat{X}_{n}=\int_{0}^{1} t \cos (2 \pi n t) b(t) d t .
\end{gathered}
$$

En resumen se tiene que

$$
I_{n}=(-1)^{n}\left[n \pi Y_{n}-n \pi \varepsilon_{n} X_{n}+2 n \pi \varepsilon_{n} \widehat{X}_{n}\right]+O\left(n^{-1+}\right)
$$

y finalmente de (12), (13), (14) y (15) se llega a

$$
\varphi_{1}^{\prime}(1)=(-1)^{n}\left[b(1)+n \pi Y_{n}-n \pi \varepsilon_{n} X_{n}+2 n \pi \varepsilon_{n} \widehat{X}_{n}+O\left(n^{-1+}\right)\right] .
$$

Por lo tanto

$$
\begin{aligned}
\varphi^{\prime}(1)= & \varphi_{0}^{\prime}(1)+\varphi_{1}^{\prime}(1)+O\left(n^{0+}\right) \\
= & (-1)^{n}\left[h-n \pi \varepsilon_{n}+\frac{n \pi \varepsilon_{n}^{3}}{6}+b(1)+n \pi Y_{n}-n \pi \varepsilon_{n} X_{n}\right] \\
& +(-1)^{n}\left[2 n \pi \varepsilon_{n} \widehat{X}_{n}+O\left(n^{-1+}\right)\right] .
\end{aligned}
$$

y en particular

$$
\varphi^{\prime}(1)=(-1)^{n}\left[h-n \pi \varepsilon_{n}+O\left(n^{0+}\right)\right] .
$$


Por otra parte, de (9) se tiene

$$
\begin{aligned}
\varphi_{0}(1) & =\frac{h}{\mu} \operatorname{sen} \mu+\cos \mu \\
& =\frac{h}{n \pi+\varepsilon_{n}} \operatorname{sen}\left(n \pi+\varepsilon_{n}\right)+\cos \left(n \pi+\varepsilon_{n}\right) \\
& =(-1)^{n}\left(\frac{h}{n \pi+\varepsilon_{n}} \operatorname{sen} \varepsilon_{n}+\cos \varepsilon_{n}\right) \\
& =(-1)^{n}\left[1+O\left(n^{-1+}\right)\right],
\end{aligned}
$$

y de (8) obtenemos también

$$
\begin{aligned}
\varphi_{1}(1) & =\frac{h}{\mu} \int_{0}^{1} \operatorname{sen} \mu(2 t-1) b(t) d t+\int_{0}^{1} \cos \mu(2 t-1) b(t) d t \\
= & \frac{h}{n \pi+\varepsilon_{n}} \int_{0}^{1} \operatorname{sen}\left(n \pi+\varepsilon_{n}\right)(2 t-1) b(t) d t \\
+ & \int_{0}^{1} \cos \left(n \pi+\varepsilon_{n}\right)(2 t-1) b(t) d \\
= & O\left(n^{-1+}\right) .
\end{aligned}
$$

Además,

$$
\varphi(1)=(-1)^{n}+O\left(n^{0+}\right) \quad \text { y la condición } \quad \varphi^{\prime}(1)=-H \varphi(1)
$$

nos lleva a

$$
(-1)^{n}\left[n \pi \varepsilon_{n}+O\left(n^{0+}\right)\right]=(-1)^{n}+O\left(n^{0+}\right),
$$

esto es $\varepsilon_{n}=O\left(n^{-1+}\right)$.

Obsérvese ahora que

$$
\begin{aligned}
\varphi_{2}(x) & =\int_{0}^{x} \frac{\operatorname{sen} \mu(x-t)}{\mu} \varphi_{1}(t) q(t) d t \\
& =\int_{0}^{x}\left[\cos \mu(x-t) \varphi_{1}(t)-\frac{\operatorname{sen} \mu(x-t)}{\mu} \varphi_{1}^{\prime}(t)\right] b(t) d t
\end{aligned}
$$

y por lo tanto

$$
\varphi_{2}^{\prime}(x)=\int_{0}^{x}\left[-\mu \operatorname{sen} \mu(x-t) \varphi_{1}(t)-\cos \mu(x-t) \varphi_{1}^{\prime}(t)\right] b(t) d t+\varphi_{1}(x) b(x)
$$

luego,

$$
\begin{aligned}
\varphi_{2}^{\prime}(1) & =\int_{0}^{1}\left[\mu \operatorname{sen} \mu(t-1) \varphi_{1}(t)-\cos \mu(t-1) \varphi_{1}^{\prime}(t)\right] b(t) d t+\varphi_{1}(1) b(1) \\
& =A-B+C,
\end{aligned}
$$


donde hemos colocado

$$
\begin{gathered}
A=\int_{0}^{1}\left[\mu \operatorname{sen} \mu(t-1) \varphi_{1}(t)\right] b(t) d t, B=\int_{0}^{1}\left[\cos \mu(t-1) \varphi_{1}^{\prime}(t)\right] b(t) d t \\
\text { y } \quad C=\varphi_{1}(1) b(1) .
\end{gathered}
$$

Antes de proceder a estimar $A, B$ y $C$, primero note que

$$
\begin{aligned}
\varphi_{1}(x)= & \frac{h}{n \pi+\varepsilon_{n}} \int_{0}^{x} \operatorname{sen}\left(\left(n \pi+\varepsilon_{n}\right)(2 t-x)\right) b(t) d t \\
& +\int_{0}^{x} \cos \left(\left(n \pi+\varepsilon_{n}\right)(2 t-x)\right) b(t) d t \\
= & \frac{h}{n \pi+\varepsilon_{n}} I_{1}+I_{2} .
\end{aligned}
$$

Dado que la primera integral de la derecha es de orden $O\left(n^{-1+}\right)$, nos concentramos en estimar el orden de la segunda integral:

$$
\begin{aligned}
I_{2}= & \int_{0}^{x} \cos \left(n \pi+\varepsilon_{n}\right)(2 t-x) b(t) d t \\
= & \int_{0}^{x} \cos \left(2 t\left(n \pi+\varepsilon_{n}\right)\right) \cos \left(x\left(n \pi+\varepsilon_{n}\right)\right) b(t) d t \\
& +\int_{0}^{x} \operatorname{sen}\left(2 t\left(n \pi+\varepsilon_{n}\right)\right) \operatorname{sen}\left(x\left(n \pi+\varepsilon_{n}\right)\right) b(t) d t \\
= & \Lambda_{1}+\Lambda_{2} .
\end{aligned}
$$

Ahora, observe que

$$
\begin{aligned}
\Lambda_{1} & =\int_{0}^{x} \cos \left(2 t\left(n \pi+\varepsilon_{n}\right)\right) \cos \left(x\left(n \pi+\varepsilon_{n}\right)\right) b(t) d t \\
& =\cos \left(\left(n \pi+\varepsilon_{n}\right) x\right) \int_{0}^{x} \cos 2 t\left(n \pi+\varepsilon_{n}\right) b(t) d t \\
& =\cos \left(\left(n \pi+\varepsilon_{n}\right) x\right) \int_{0}^{x}\left[\cos (2 \pi n t) \cos \left(2 \varepsilon_{n} t\right)-\operatorname{sen}(2 \pi n t) \operatorname{sen}\left(2 \varepsilon_{n} t\right)\right] b(t) d t
\end{aligned}
$$

Note que

$$
\begin{aligned}
\int_{0}^{x} \cos (2 \pi n t) \cos \left(2 \varepsilon_{n} t\right) b(t) d t & =\int_{0}^{x} \cos (2 \pi n t)\left(1+O\left(\frac{1}{n^{2}}\right)\right) b(t) d t \\
& =\int_{0}^{x} \cos (2 \pi n t) b(t) d t+O\left(\frac{1}{n^{2}}\right) .
\end{aligned}
$$


y entonces se sigue que

$$
\begin{aligned}
& \Lambda_{1}=\cos \left(\left(n \pi+\varepsilon_{n}\right) x\right)\left[\int_{0}^{x} \cos (2 \pi n t) b(t) d t-2 \varepsilon_{n} \int_{0}^{x} t \operatorname{sen}(2 \pi n t) b(t) d t\right] \\
& \quad+O\left(\frac{1}{n^{2}}\right) .
\end{aligned}
$$

Cálculos similares demuestran que

$$
\begin{aligned}
\Lambda_{2}= & \operatorname{sen}\left(\left(n \pi+\varepsilon_{n}\right) x\right) \int_{0}^{x} \operatorname{sen}(2 \pi n t) b(t) d t+ \\
& \varepsilon_{n} \operatorname{sen}\left(\left(n \pi+\varepsilon_{n}\right) x\right) \int_{0}^{x} t \cos (2 \pi n t) b(t) d t+O\left(n^{-2+}\right) .
\end{aligned}
$$

Usando el Corolario 1 de [3] es fácil ver que $\Lambda_{1}=O\left(n^{-1+}\right)$, y similarmente que $\Lambda_{2}=O\left(n^{-1+}\right)$, por lo que se obtiene que $I_{2}=O\left(n^{-1+}\right)$, y análogamente se sigue que $I_{1}=O\left(n^{-1+}\right)$, y entonces

$$
\begin{aligned}
& \varphi_{1}(x)= O\left(n^{-2+}\right)+\cos \left(\left(n \pi+\varepsilon_{n}\right) x\right) \int_{0}^{x} \cos (2 \pi n t) b(t) d t \\
&-2 \varepsilon_{n} \cos \left(\left(n \pi+\varepsilon_{n}\right) x\right) \int_{0}^{x} t \operatorname{sen}(2 \pi n t) b(t) d t \\
&+\operatorname{sen}\left(\left(n \pi+\varepsilon_{n}\right) x\right) \int_{0}^{x} \operatorname{sen}(2 \pi n t) b(t) d t \\
&+\varepsilon_{n} \operatorname{sen}\left(\left(n \pi+\varepsilon_{n}\right) x\right) \int_{0}^{x} t \cos (2 \pi n t) b(t) d t \\
&= O\left(n^{-2+}\right)+\cos \left(\left(n \pi+\varepsilon_{n}\right) x\right) \Omega_{1}+\operatorname{sen}\left(\left(n \pi+\varepsilon_{n}\right) x\right) \Omega_{2}, \\
& \Omega_{1}=\left[\int_{0}^{x} \cos (2 \pi n t) b(t) d t-2 \varepsilon_{n} \int_{0}^{x} t \operatorname{sen}(2 \pi n t) b(t) d t\right] \\
&=X_{n}(x)-2 \varepsilon_{n} \widehat{Y}_{n}(x)
\end{aligned}
$$

en donde hemos colocado

$$
\begin{aligned}
\Omega_{2} & =\left[\int_{0}^{x} \operatorname{sen}(2 \pi n t) b(t) d t+\varepsilon_{n} \int_{0}^{x} t \cos (2 \pi n t) b(t) d t\right] \\
& =X_{n}(x)-2 \varepsilon_{n} \widehat{Y}_{n}(x) .
\end{aligned}
$$


VALORES PROPIOS ASOCIADOS AL OPERADOR DE HILL UNIDIMENSIONAL

Regresando a (17), y luego de algunas simplificaciones, se sigue que

$$
\begin{aligned}
A= & \int_{0}^{1} \mu \operatorname{sen} \mu(t-1) \varphi_{1}(t) b(t) d t \\
= & (-1)^{n} n \pi\left\{\int_{0}^{1} \operatorname{sen}\left(n \pi t+\varepsilon_{n}(t-1)\right)\left[\cos \left(\left(n \pi+\varepsilon_{n}\right) t\right) \Omega_{1}\right] b(t) d t\right\} \\
& +(-1)^{n} n \pi\left\{\int_{0}^{1}\left[\operatorname{sen}\left(n \pi t+\varepsilon_{n}(t-1)\right) \operatorname{sen}\left(\left(n \pi+\varepsilon_{n}\right) t\right) \Omega_{2}\right] b(t) d t\right\} \\
& +O\left(n^{-1+}\right) \\
= & \frac{(-1)^{n}}{4} \int_{0}^{1} b^{2}(u) d u+O\left(n^{-1+}\right)
\end{aligned}
$$

en donde se ha utilizado el hecho que

$$
\begin{aligned}
\frac{1}{2} \int_{0}^{1} Y_{n}(t) b(t) d t & =\frac{1}{2} \int_{0}^{1}\left[-\frac{b(t)}{2 \pi n} \cos (2 \pi n t)+O\left(n^{-2+}\right)\right] b(t) d t \\
& =\frac{-1}{4 \pi n} \int_{0}^{1} b^{2}(t) \cos (2 \pi n t) d t+O\left(n^{-2+}\right) \\
& =O\left(n^{-2+}\right)
\end{aligned}
$$

por Borel-Cantelli y desigualdades de martingalas. Además,

$$
\begin{aligned}
Y_{n}(t) & =\int_{0}^{t} b(s) \operatorname{sen}(2 \pi n s) d s \\
& =\frac{1}{2 \pi n}\left[\int_{0}^{t} \cos (2 \pi n s) d b(s)-b(t) \cos (2 \pi n t)\right] \\
& =-\frac{b(t)}{2 \pi n} \cos (2 \pi n t)+O\left(n^{-2+}\right)
\end{aligned}
$$

así que,

$$
\begin{aligned}
\int_{0}^{t} b(u) \cos (2 \pi n u) Y_{n}(u) d u & =-\int_{0}^{t} \frac{b^{2}(u)}{2 \pi n} \cos ^{2}(2 \pi n u) d u+O\left(n^{-2+}\right) \\
& =-\frac{1}{4 \pi n} \int_{0}^{t} b^{2}(u) d u+O\left(n^{-2+}\right)
\end{aligned}
$$

De manera completamente análoga se tiene que

$$
\int_{0}^{t} b(u) \operatorname{sen}(2 \pi n u) X_{n}(u) d u=\frac{1}{4 \pi n} \int_{0}^{t} b^{2}(u) d u+O\left(n^{-2+}\right) .
$$


Por su parte

$$
B=\int_{0}^{1}\left[\cos \mu(t-1) \varphi_{1}^{\prime}(t)\right] b(t) d t
$$

en donde luego de unos cálculos se concluye que

$$
\begin{aligned}
\varphi_{1}^{\prime}(x)= & -h \int_{0}^{x} \cos \left(\left(n \pi \varepsilon_{n}\right)(2 t-x)\right) b(t) d t+\frac{h}{n \pi \varepsilon_{n}} \operatorname{sen}\left(n \pi+\varepsilon_{n}\right) b(x) \\
& +\int_{0}^{x}\left(n \pi+\varepsilon_{n}\right) \operatorname{sen}\left(\left(n \pi \varepsilon_{n}\right)(2 t-x)\right) b(t) d t+\cos \left(\left(n \pi+\varepsilon_{n}\right) x\right) b(x) \\
= & n \pi Y_{n}(x) \cos (n \pi x)-n \pi X_{n}(x) \operatorname{sen}(n \pi x)+b(x) \cos (n \pi x)+O\left(n^{-1+}\right)
\end{aligned}
$$

y por lo tanto

$$
\begin{aligned}
B= & \int_{0}^{1}\left[\cos \mu(t-1) \varphi_{1}^{\prime}(t)\right] b(t) d t \\
= & \int_{0}^{1} \cos \left[\left(n \pi+\varepsilon_{n}\right)(t-1)\right]\left[n \pi Y_{n}(t) \cos (n \pi t)-n \pi X_{n}(t) \operatorname{sen}(n \pi t)\right] \\
& \times b(t) d t+\int_{0}^{1} \cos \left[\left(n \pi+\varepsilon_{n}\right)(t-1)\right][b(t) \cos (n \pi t)] b(t) d t+O\left(n^{-1+}\right) \\
= & \int_{0}^{1} \cos [n \pi(t-1)]\left[n \pi Y_{n}(t) \cos (n \pi t)-n \pi X_{n}(t) \operatorname{sen}(n \pi t)\right] b(t) d t \\
& +\int_{0}^{1} \cos [n \pi(t-1)][b(t) \cos (n \pi t)] b(t) d t+O\left(n^{-1+}\right) \\
= & (-1)^{n}\left[\int_{0}^{1} n \pi Y_{n}(t) \cos ^{2}(n \pi t) b(t) d t-\int_{0}^{1} n \pi X_{n}(t) \operatorname{sen}(n \pi t)\right. \\
& \left.\times \cos (n \pi t) b(t) d t+\int_{0}^{1} b^{2}(t) \cos ^{2}(n \pi t)+O\left(n^{-1+}\right)\right]
\end{aligned}
$$

Intentaremos ahora hallar una expresión más simple para $B$. Sean:

$$
\begin{aligned}
& \beta_{1}=\int_{0}^{1} n \pi Y_{n}(t) \cos ^{2}(n \pi t) b(t) d t \\
& \beta_{2}=n \pi \int_{0}^{1} X_{n}(t) \operatorname{sen}(n \pi t) \cos (n \pi t) b(t) d t \\
& \beta_{3}=\int_{0}^{1} b^{2}(t) \cos ^{2}(n \pi t) d t
\end{aligned}
$$


Entonces por (20) y (21) se tienen las siguientes estimaciones:

$$
\begin{aligned}
\beta_{1} & =\int_{0}^{1} n \pi Y_{n}(t) \cos ^{2}(n \pi t) b(t) d t \\
& =\frac{n \pi}{2} \int_{0}^{1} Y_{n}(t) b(t) d t-\frac{n \pi}{2} \int_{0}^{1} Y_{n}(t) \cos (2 \pi n t) b(t) d t \\
& =\frac{n \pi}{2} \cdot O\left(n^{-2+}\right)-\frac{n \pi}{2}\left[\frac{-1}{4 \pi n} \int_{0}^{1} b^{2}(t) d t+O\left(n^{-2+}\right)\right] \\
& =\frac{1}{8} \int_{0}^{1} b^{2}(t) d t+O\left(n^{-1+}\right),
\end{aligned}
$$

por su parte

$$
\begin{aligned}
\beta_{2} & =n \pi \int_{0}^{1} X_{n}(t) \operatorname{sen}(n \pi t) \cos (n \pi t) b(t) d t \\
& =\frac{n \pi}{2} \int_{0}^{1} X_{n}(t) \operatorname{sen}(2 n \pi t) b(t) \\
& =\frac{n \pi}{2} \int_{0}^{1}\left[\frac{b^{2}(t)}{2 \pi n} \operatorname{sen}^{2}(2 \pi n t)\right] d t+O\left(n^{-1+}\right) \\
& =\frac{n \pi}{2 \cdot 2 \pi n \cdot 2} \int_{0}^{1} b^{2}(t)[1-\cos (4 \pi n t)] d t+O\left(n^{-1+}\right) \\
& =\frac{1}{8} \int_{0}^{1} b^{2}(t) d t+O\left(n^{-1+}\right)
\end{aligned}
$$

y finalmente,

$$
\begin{aligned}
\beta_{3}=\int_{0}^{1} b^{2}(t) \cos ^{2}(n \pi t) d t & =\frac{1}{2} \int_{0}^{1} b^{2}(t) d t+\frac{1}{2} \int_{0}^{1} b^{2}(t) \cos (2 \pi n t) d t \\
& =\frac{1}{2} \int_{0}^{1} b^{2}(t) d t+O\left(n^{-2+}\right) .
\end{aligned}
$$

Luego,

$$
\begin{aligned}
B & =(-1)^{n}\left(\beta_{1}-\beta_{2}+\beta_{3}\right) \\
& =(-1)^{n}\left[\frac{1}{8} \int_{0}^{1} b^{2}(t) d t-\frac{1}{8} \int_{0}^{1} b^{2}(t) d t+O\left(n^{-1+}\right)+\frac{1}{2} \int_{0}^{1} b^{2}(t) d t\right] .
\end{aligned}
$$


Por último, tenemos que $C=\varphi_{1}(1) b(1)=O\left(n^{-1+}\right)$, de donde se obtiene por (16) que

$$
\begin{aligned}
\varphi_{2}^{\prime}(1) & =A-B+C \\
& =\frac{(-1)^{n}}{4} \int_{0}^{1} b^{2}(u) d u+\frac{(-1)^{n+1}}{2} \int_{0}^{1} b^{2}(t) d t+O\left(n^{-1+}\right) \\
& =-\frac{(-1)^{n}}{4} \int_{0}^{1} b^{2}(t) d t+O\left(n^{-1+}\right) .
\end{aligned}
$$

De (10)

$$
\varphi_{0}^{\prime}(1)+\varphi_{1}^{\prime}(1)+\varphi_{2}^{\prime}(1)=-H\left[\varphi_{0}(1)+\varphi_{1}(1)+\varphi_{2}(1)+O\left(\mu^{-1+}\right)\right]
$$

es decir

$$
\begin{aligned}
\varphi^{\prime}(1)= & (-1)^{n}\left[h-n \pi \varepsilon_{n}+\frac{b(1)}{2}-n \pi \varepsilon_{n} X_{n}(1)+2 n \pi \varepsilon_{n} \widehat{X}_{n}+\varphi_{2}^{\prime}(1)\right] \\
& +O\left(n^{-1+}\right)
\end{aligned}
$$

con

$$
\begin{gathered}
\varphi_{0}^{\prime}(1)=(-1)^{n}\left[h-n \pi \varepsilon_{n}+O\left(n^{-1+}\right)\right], \quad \varphi_{2}^{\prime}(1)=-\frac{(-1)^{n}}{4} \int_{0}^{1} b^{2}(t) d t+O\left(n^{-1+}\right), \\
\varphi_{0}(1)=(-1)^{n}\left[1+O\left(n^{-1+}\right)\right] \quad \text { y } \quad \varphi_{1}(1)=O\left(n^{-1+}\right) .
\end{gathered}
$$

Note que

$$
n \pi Y_{n}(1)=-\frac{1}{2} b(1)
$$

y entonces

$$
\begin{aligned}
\varphi^{\prime}(1)= & (-1)^{n}\left[h-n \pi \varepsilon_{n}+\frac{b(1)}{2}-n \pi \varepsilon_{n} X_{n}(1)+2 n \pi \varepsilon_{n} \widehat{X}_{n}\right. \\
& \left.-\frac{(-1)^{n}}{4} \int_{0}^{1} b^{2}(t) d t+O\left(n^{-1+}\right)\right] .
\end{aligned}
$$

donde

$$
n \pi \varepsilon_{n} X_{n}(1)=O\left(n^{-1+}\right), \quad 2 n \pi \varepsilon_{n} \widehat{X}_{n}=O\left(n^{-1+}\right) .
$$

Por lo tanto, de (22) se sigue que

$$
h-n \pi \varepsilon_{n}+\frac{b(1)}{2}-\frac{1}{4} \int_{0}^{1} b^{2}(t) d t+O\left(n^{-1+}\right)=-H+O\left(n^{-1+}\right),
$$

lo que nos da al despejar

$$
\varepsilon_{n}=\frac{h+H+\frac{b(1)}{2}}{n \pi}-\frac{1}{4 n \pi} \int_{0}^{1} b^{2}(t) d t+O\left(n^{-2+}\right) .
$$


Finalmente, al utilizar esta aproximación para $\varepsilon_{n}$ se obtiene el desarrollo asintótico de $\lambda_{n}$,

$$
\begin{aligned}
\lambda_{n} & =n^{2} \pi^{2}+2 n \pi \varepsilon_{n}+\varepsilon_{n}^{2} \\
& =n^{2} \pi^{2}+2 h+2 H+b(1)-\frac{1}{2} \int_{0}^{1} b^{2}(t) d t+O\left(n^{-1+}\right)
\end{aligned}
$$

lo que completa la demostración.

Compárese la similitud de este resultado con el de [3], en donde se obtiene la sucesión de valores propios

$$
\lambda_{n}=n^{2} \pi^{2}+b(1)-\int_{0}^{1} \cos (2 n \pi t) d b(t)+O\left(n^{-1+}\right)
$$

para valores grandes de $n$, con $b$ el movimiento browniano correspondiente.

El caso particular $H=h=0$, corresponde a las denominadas condiciones de Neumann. En el caso general es posible intentar obtener una mejor aproximación de los valores propios, pero es claro que los cálculos tienden a complicarse un poco.

\section{Referencias}

[1] Ahlfors, L.V. (1979) Complex Analysis: an Introduction to the Theory of Analytic Functions of One Variable. Tercera edición, McGraw-Hill, New York.

[2] Breiman, L. (1992) Probability. Classics in Applied Mathematics, SIAM.

[3] Cambronero, S.; McKean, H.P. (1995) "Valores propios de Dirichlet asociados a la ecuación de Hill con potencial de ruido blanco", Revista de Matemática: Teoría y Aplicaciones 2(2): 1-7.

[4] Cambronero, S.; McKean, H.P.: (1999) "The ground eigenvalue of Hill's equation with white noise potential", Comm. in Pure and Applied Mathematics LII: 1277-1294

[5] Cambronero, S. (1996) "La ecuación de Hill con potencial irregular", Revista de Matemática: Teoría y Aplicaciones 3(1): 25-34.

[6] Cambronero, S. (1997) "La ecuación de Hill y el movimiento browniano circular", $V$ Encuentro Centroamericano de Investigadores en Matemática (ECADIM): 133-140.

[7] Cambronero, S. (1997) "Algunos potenciales del tipo Ornstein-Uhlenbeck para el operador de Schrödinger", Revista de Matemática: Teoría y Aplicaciones 4(1).

[8] Chung, K.L. (1974) A Course in Probability Theory. Academic Press, Inc. Second ed.

[9] Doob, J.L. (1953) Stochastic Processes. John Wiley and Sons, New York,.

[10] Eastham, M.S.P. (1971) The Spectral Theory of Periodic Differential Equations. Scottish Academic Press.

[11] Hochstadt, H. (1961) "Asymptotic estimates for the Sturm - Liouville spectrum", Com. Pure Appl. 14: 749-764. 
[12] Karatzas, I.; Shreve, S.E. (1991) Brownian Motion and Stochastic Calculus. SpringerVerlag. Segunda edición.

[13] Lax, M.; Phillips, J. C. (1958) "One-dimensional impurity bands", Phys. Review 110(1).

[14] Levitan, B.M.; Sargsjar, I.S. (1991) Sturm-Liouville and Dirac Operators. Kluwer Academic Publishers.

[15] McKean, H. (1969) Stochastic Integrals. Academic Press, New York.

[16] McKean, H.; Moerbeke, P. (1975) "The spectrum of Hill's equation", Inv. Math. 30: $217-274$.

[17] Schmidt, H. (1957) "Disordered one-dimensional crystals", Phys. Review. 105(2). 